\title{
The Distribution of the Ratio of the Products of Two Independent $\alpha-\mu$ Variates and Its Application in the Performance Analysis of Relaying Communication Systems
}

\author{
Ana Matovic,, ${ }^{1}$ Edis Mekić, ${ }^{2}$ Nikola Sekulović, ${ }^{3}$ Mihajlo Stefanović, ${ }^{1}$ \\ Marija Matović, ${ }^{1}$ and Časlav Stefanović ${ }^{4}$ \\ ${ }^{1}$ Faculty of Electronic Engineering, University of Niš, 18000 Niš, Serbia \\ ${ }^{2}$ State University of Novi Pazar, 36300 Novi Pazar, Serbia \\ ${ }^{3}$ The School of Higher Technical Proffesional Education, 18000 Niš, Serbia \\ ${ }^{4}$ Faculty of Natural Sciences and Mathematics, University of Priština, 38220 Kosovska Mitrovica, Serbia \\ Correspondence should be addressed to Edis Mekić; emekic@np.ac.rs
}

Received 9 January 2013; Revised 24 February 2013; Accepted 24 February 2013

Academic Editor: Zheng-Guang Wu

Copyright (C) 2013 Ana Matović et al. This is an open access article distributed under the Creative Commons Attribution License, which permits unrestricted use, distribution, and reproduction in any medium, provided the original work is properly cited.

We present novel general, simple, exact, and closed-form expressions for the probability density function (PDF) and cumulative distribution function (CDF) of the ratio of the products of two independent $\alpha-\mu$ variates, where all variates have identical values of alpha parameter. Obtained results are applied in analysis of multihop wireless communication systems in different fading transmission environments. The proposed theoretical analysis is also complemented by various graphically presented numerical results.

\section{Introduction}

Modern technology is characterized by increased need for mobile computing and communications devices. This demand for mobility led to the introduction of number of different wireless networking technologies such as wireless local area networks, Bluetooth technologies, ultrawideband networks, mobile ad hoc networks, and Wi-max [1-5]. All those technologies are based on relaying techniques which enable network connectivity where traditional architectures are impractical due to location constraints.

Since all those technologies are in essence wireless communication systems, one of occurring problems for this type of system is fading caused by multipath propagation. When a received signal experiences fading during transmission, signal envelope fluctuates over time [6]. In addition to fading, the wireless transmission can also be subjected to shadowing which is the result of large obstacles and deviations in terrain profile between transmitter and receiver. In such composite environments, signal envelope can be modeled with product of two random variables $[7,8]$.
Besides fading another important aspect of this type of communication system is cochannel interference (CCI). CCI is a result of frequency reuse which is essential in increasing system capacity and is statistically modeled with ratio of random variables [9].

Wide range of statistical models exists for describing the statistical behavior of signal envelope whose accuracy and veracity depend on communication scenario and propagation environment. The most frequently applied models in the technical literature are Rayleigh, Nakagami- $m$, Weibull and $\alpha-\mu$. Since $\alpha-\mu$ variate comprise, Rayleigh, Nakagami$m$, Weibull variates as special cases. Once we derived $\alpha$ $\mu$ distribution, the special cases can be obtained by the appropriate setting of the parameters $\alpha$ and $\mu$.

The Rayleigh model is frequently used to describe multipath fading with no direct line-of-sight (LOS) path and where a number of objects affect signal like high density city areas $[10,11]$.

The Weibull distribution exhibits an excellent fit to experimental fading channel measurements, for both indoor [12] 
and outdoor [13] environments. Also good results are provided in urban environments. Fading severity in the Weibull fading environment is described by Weibull parameter $\beta$; as these parameters increase, fading severity decreases.

The Nakagami- $m$ distribution has gained widespread application in the modeling of physical radio channels since it shows great agreement with experimentally obtained results [14]. Fading severity in Nakagami- $m$ environments is described by Nakagami parameter $m$, respectively. As these parameters increase, fading severity decreases [15].

The $\alpha-\mu$ distribution is a general gamma distribution that can be used to better represent the small-scale variation of the fading signal [16]. Parameter $\mu$ is associated with the number of multipath clusters where all dissipated waves have same powers. Parameter $\alpha$ is related to the nonlinearity of the environment as the module of power [17].

These different ratios of variates and products of variates were used as starting point for analysis of the performance of wireless telecommunication systems $[18,19]$.

Main contribution of this paper is the derivation of useful closed-form expressions for the probability density function (PDF) and cumulative distribution function (CDF) of ratio of product of two random variables. These expressions can be used as general theoretical tool in the performance analysis of wireless communication systems in realistic scenario in modern urban areas where the product of random variables in nominator can represent signal which suffers from fading and shadowing while the random variable in denominator can represent CCI also propagating thoughts fading and shadowing environment.

Therefore, the ratio of such random variables is signal-tointerference ratio (SIR). Rayleigh, Nakagami- $m$, Weibull, and $\alpha-\mu$ distributions are included in our analysis to give insight in a wide range of usages of results presented in the paper, that is, to model different transmission environments.

Capitalizing on closed-form expressions derived in the paper in terms of Meijer G functions, the outage probability of multihop system is determined and discussed as an important and widely accepted performance measure. Multihop is a technique by which the channel from the source $(S)$ to the destination (D) is split into several, possibly shorter, links using relays $\left(R_{i}, i=\overline{1, N-1}\right)$.

\section{Statistics of Ratio of Products of Two Random Variables}

First, we present different distributions describing statistical behavior of arbitrary random variable $\gamma$ which are in cases of
Rayleigh, Weibull, Nakagami- $m$, and $\alpha-\mu$ distributions given by respected distribution.

In the case of Rayleigh distribution random variable is given by

$$
p_{\gamma}(\gamma)=\frac{2}{\Omega_{\gamma}} \gamma e^{-\left(\gamma^{2} / \Omega_{\gamma}\right)}
$$

where $\Omega_{\gamma}=E\left\langle\gamma^{2}\right\rangle$ and $E\langle\cdot\rangle$ denotes expectation.

In the case of Weibull distribution random variable is given by

$$
p_{\gamma}(\gamma)=\frac{\beta}{\Omega_{\gamma}} \gamma^{\beta-1} e^{-\left(\gamma^{\beta} / \Omega_{\gamma}\right)}
$$

where $\Omega_{\gamma}=E\left\langle\gamma^{\beta}\right\rangle$ and $E\langle\cdot\rangle$ denotes expectation.

In the case of Nakagami- $m$ distribution random variable is given by

$$
p_{\gamma}(\gamma)=\frac{2 m_{\gamma}^{m_{\gamma}}}{\Gamma\left(m_{\gamma}\right) \Omega_{\gamma}^{m_{\gamma}}} \gamma^{2 m_{\gamma}-1} e^{-\left(m_{\gamma} \gamma^{2} / \Omega_{\gamma}\right)},
$$

where $\Omega_{\gamma}=E\left\langle\gamma^{2}\right\rangle, \Gamma(\cdot)$ is gamma function, and $m_{\gamma}$ is Nakagami- $m$ parameters that range from 0.5 to $\infty$.

In the case of $\alpha-\mu$ distributed random variables are described with the following equations:

$$
p_{\gamma}(\gamma)=\alpha\left(\frac{\mu_{\gamma}}{\Omega_{\gamma}}\right)^{\mu_{\gamma}} \frac{\gamma^{\alpha \mu_{\gamma}-1}}{\Gamma\left(\mu_{\gamma}\right)} e^{-\left(\mu_{\gamma} \gamma^{\alpha} / \Omega_{\gamma}\right)}
$$

where $\Omega_{\gamma}=E\left\langle\gamma^{\alpha}\right\rangle, \alpha>0$ is parameter related to the nonlinearity, and $\mu_{\gamma}$ is the inverse of the normalized variance of $\gamma^{\alpha},\left(\mu_{\gamma} \geq 0.5\right)$. The $\alpha-\mu$ distribution is a general distribution that includes as special cases Nakagami- $m$ distribution for $\alpha=2$ and Weibull distribution for $\mu=1$. In all those cases value of $\Omega_{\gamma}>0$ since they represent realistic values of the power signal and interference.

In this section, closed-form expressions for the PDF and $\mathrm{CDF}$ of ratio of random variable and product of two random variables, $\lambda=x y / z w$ (where values of the random variables $x, y, z$, and $w$ are defined by including these variables in expressions (1), (2), (3), and (4), are obtained using solutions for PDF of $\alpha-\mu$ variable $t=x y / z$ derived in [14]:

$$
\begin{aligned}
p_{t}(t)= & \alpha\left(\frac{\mu_{x} \mu_{z}}{\mu_{y}}\right)^{(1 / 2)\left(\mu_{x}+\mu_{z}-1\right)}\left(\frac{\Omega_{y}}{\Omega_{x} \Omega_{z}}\right)^{(1 / 2)\left(\mu_{x}+\mu_{z}-1\right)} t^{(\alpha / 2)\left(\mu_{x}+\mu_{z}-1\right)-1} \\
& \times \frac{1}{\Gamma\left(\mu_{x}\right) \Gamma\left(\mu_{y}\right) \Gamma\left(\mu_{z}\right)} G_{1,2}^{2,1}\left(\begin{array}{l|c}
\frac{\mu_{x} \mu_{z}}{\mu_{y}} \frac{\Omega_{y}}{\Omega_{x} \Omega_{z}} t^{\alpha} & -\frac{1}{2}\left(\mu_{x}+2 \mu_{y}+\mu_{z}-3\right) \\
\frac{1}{2}\left(\mu_{z-} \mu_{x}+1\right), \frac{1}{2}\left(1-\mu_{z+} \mu_{x}\right)
\end{array}\right),
\end{aligned}
$$


and applying

$$
\begin{gathered}
p_{\lambda}(\lambda)=\int_{0}^{\infty}|J| p_{t}(\lambda w) p_{w}(w) d w \\
|J|=\left|\frac{d t}{d \lambda}\right|=w .
\end{gathered}
$$

CDF of $\lambda$ can be obtained by definition as

$$
F_{\lambda}(\lambda)=\int_{0}^{\lambda} p_{\lambda}(s) d s
$$

After applying the described procedure, with the aid of [18, equations (2.8), (2.9), (2.10), and (2.11)], [20, equations (3.461), (6.631,(3)), and (7.813 (1))]], [21], and [22, equation (26)], the PDFs and CDFs of $\lambda$ in different scenarios can be expressed in terms of Meijer $\mathrm{G}$ functions. (a) Rayleigh scenario:

$$
\begin{gathered}
p_{\lambda}(\lambda)=2 \sqrt{\frac{\Omega_{z} \Omega_{w}}{\Omega_{x} \Omega_{y}}} G_{2,2}^{2,2}\left(\begin{array}{l|l}
\frac{\Omega_{z} \Omega_{w}}{\Omega_{x} \Omega_{y}} \lambda^{2} & -\frac{1}{2},-\frac{1}{2} \\
\frac{1}{2}, \frac{1}{2}
\end{array}\right), \\
F_{\lambda}(\lambda)=2 \sqrt{\frac{\Omega_{z} \Omega_{w}}{\Omega_{x} \Omega_{y}} \lambda G_{3,3}^{2,3}}\left(\begin{array}{l|l}
\frac{\Omega_{z} \Omega_{w}}{\Omega_{x} \Omega_{y}} \lambda^{2} & -\frac{1}{2},-\frac{1}{2}, \frac{1}{2} \\
\frac{1}{2}, \frac{1}{2},-\frac{1}{2}
\end{array}\right) .
\end{gathered}
$$

(b) Weibull scenario:

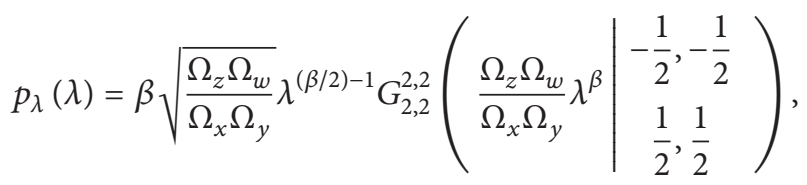

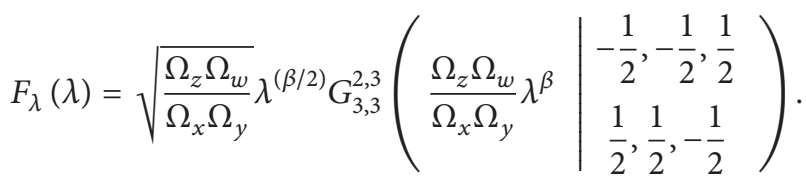

(c) Nakagami-m scenario:

$$
\begin{aligned}
& p_{\lambda}(\lambda)= 2\left(\frac{m_{x} m_{y}}{m_{z} m_{w}}\right)^{(1 / 2)\left(m_{x}+m_{y}-1\right)} \\
& \times\left(\frac{\Omega_{z} \Omega_{w}}{\Omega_{x} \Omega_{y}}\right)^{(1 / 2)\left(m_{x}+m_{y}-1\right)} \frac{1}{\Gamma\left(m_{x}\right) \Gamma\left(m_{y}\right) \Gamma\left(m_{z}\right) \Gamma\left(m_{w}\right)} \lambda^{m_{x}+m_{y}-2} \\
& \times G_{2,2}^{2,2}\left(\frac{m_{x} m_{y}}{m_{z} m_{w}} \frac{\Omega_{z} \Omega_{w}}{\Omega_{x} \Omega_{y}} \lambda^{2} \mid-\frac{1}{2}\left(m_{x}+m_{y}+2 m_{w}-3\right),-\frac{1}{2}\left(m_{x}+2 m_{z}+m_{y}-3\right)\right), \\
& F_{\lambda}(\lambda)=\left.\left.\frac{m_{x} m_{y}}{m_{z} m_{w}}\right)^{(1 / 2)\left(m_{x}+m_{y}-1\right)}\left(\frac{\left.\Omega_{z}-m_{x}+1\right), \frac{1}{2}\left(1-m_{y}+m_{x}\right)}{\Omega_{x} \Omega_{y}}\right)^{(1 / 2)\left(m_{x}+m_{y}-1\right)} \frac{1}{\Gamma\left(m_{x}\right) \Gamma\left(m_{y}\right) \Gamma\left(m_{z}\right) \Gamma\left(m_{w}\right)}\right), \\
&\left.\times \lambda^{m_{x}+m_{y}-1}\right) \\
& \times G_{3,3}^{2,3}\left(\frac{m_{x} m_{y}}{m_{z} m_{w}} \frac{\Omega_{z} \Omega_{w}}{\Omega_{x} \Omega_{y}} \lambda^{2} \mid-\frac{1}{2}\left(m_{x}+m_{y}+2 m_{w}-3\right),-\frac{1}{2}\left(m_{x}+2 m_{z}+m_{y}-3\right), \frac{1}{2}\left(3-m_{x}-m_{y}\right)\right. \\
& \frac{1}{2}\left(m_{y}-m_{x}+1\right), \frac{1}{2}\left(1-m_{y}+m_{x}\right), \frac{1}{2}\left(1-m_{x}-m_{y}\right)
\end{aligned}
$$

(d) $\alpha-\mu$ scenario:

$$
\begin{aligned}
p_{\lambda}(\lambda)= & \alpha\left(\frac{\mu_{x} \mu_{y}}{\mu_{z} \mu_{w}}\right)^{(1 / 2)\left(\mu_{x}+\mu_{y}-1\right)}\left(\frac{\Omega_{z} \Omega_{w}}{\Omega_{x} \Omega_{y}}\right)^{(1 / 2)\left(\mu_{x}+\mu_{y}-1\right)} \lambda^{(\alpha / 2)\left(\mu_{x}+\mu_{y}-1\right)-1} \frac{1}{\Gamma\left(\mu_{x}\right) \Gamma\left(\mu_{y}\right) \Gamma\left(\mu_{z}\right) \Gamma\left(\mu_{w}\right)} \\
& \times G_{2,2}^{2,2}\left(\frac{\mu_{x} \mu_{y}}{\mu_{z} \mu_{w}} \frac{\Omega_{z} \Omega_{w}}{\Omega_{x} \Omega_{y}} \lambda^{\alpha} \mid \begin{array}{r}
-\frac{1}{2}\left(\mu_{x}+\mu_{y}+2 \mu_{w}-3\right),-\frac{1}{2}\left(\mu_{x}+2 \mu_{y}+\mu_{z}-3\right) \\
\frac{1}{2}\left(\mu_{y}-\mu_{x}+1\right), \frac{1}{2}\left(1-\mu_{y}+\mu_{x}\right)
\end{array}\right),
\end{aligned}
$$




$$
\begin{aligned}
& F_{\lambda}(\lambda)=\alpha\left(\frac{\mu_{x} \mu_{y}}{\mu_{z} \mu_{w}}\right)^{(1 / 2)\left(\mu_{x}+\mu_{y}-1\right)}\left(\frac{\Omega_{z} \Omega_{w}}{\Omega_{x} \Omega_{y}}\right)^{(1 / 2)\left(\mu_{x}+\mu_{y}-1\right)} \frac{1}{\Gamma\left(\mu_{x}\right) \Gamma\left(\mu_{y}\right) \Gamma\left(\mu_{z}\right) \Gamma\left(\mu_{w}\right)} \\
& \times \lambda^{(\alpha / 2)\left(\mu_{x}+\mu_{y}-1\right)} G_{3,3}^{2,3}\left(\begin{array}{c|c}
\frac{\mu_{x} \mu_{y}}{\mu_{z} \mu_{w}} \frac{\Omega_{z} \Omega_{w}}{\Omega_{x} \Omega_{y}} \lambda^{\alpha} & -\frac{1}{2}\left(\mu_{x}+\mu_{y}+2 \mu_{w}-3\right),-\frac{1}{2}\left(\mu_{x}+2 \mu_{y}+\mu_{z}-3\right), \frac{1}{2}\left(3-\mu_{x}-\mu_{y}\right) \\
\frac{1}{2}\left(\mu_{y}-\mu_{x}+1\right), \frac{1}{2}\left(1-\mu_{y}+\mu_{x}\right), \frac{1}{2}\left(1-\mu_{x}-\mu_{y}\right)
\end{array}\right) .
\end{aligned}
$$

Using expression (11) we have calculated CDF and PDF for the ratio of products of two $\alpha-\mu$ distributions with special case of Nakagami- $m$ in Figure 1.

\section{An Application of Presented Theoretical Results in Wireless Communication Systems: The Performance Analysis of Multihop Systems}

The product of random variables, $x_{i} y_{i}$, represents signal envelope which suffers from fading and shadowing while the random variable $z_{i} w_{i}$ represents CCI envelope at the input of $i$ th terminal $(i=\overline{1, N})$. The random variable $\lambda_{i}=x_{i} z_{i} / y_{i} w_{i}$ presents SIR value at the input of $i$ th terminal.

Widely accepted system performance indicator is outage probability defined as probability of having SIR value lower than predetermined threshold $\lambda_{0}$ which defines required QoS [23-25]. The system failure can occur in sections $S-$ $\mathrm{R}_{1}, \mathrm{R}_{1}-\mathrm{R}_{2}, \mathrm{R}_{2}-\mathrm{R}_{3}, \ldots, \mathrm{R}_{j-1}-\mathrm{R}_{j}, \ldots, \mathrm{R}_{N-1}$-D when some of the values of $\lambda_{1}, \lambda_{2}, \lambda_{3}, \ldots, \lambda_{j}, \ldots, \lambda_{N}$ are below the predetermined threshold $\lambda_{0}$. Deriving PDF of minimum of $\lambda_{i}$, $\lambda=\min \left\{\lambda_{1}, \lambda_{2}, \ldots \lambda_{N}\right\}$, is important for analyzing multihop relayed communication systems in which the source terminal communicates with the destination terminal through a number of relay terminals. It can be obtained based on previous results as $\lambda=\min \left(\lambda_{1}, \ldots, \lambda_{j} \ldots, \lambda_{N}\right)$

$$
p_{\lambda}(\lambda)=\sum_{N}^{n=1} p_{\lambda_{n}}(\lambda) \prod_{\substack{k=1 \\ k \neq n}}^{N}\left(1-F_{\lambda_{k}}(\lambda)\right)
$$

The outage probability of multihop system is defined as

$$
P_{\text {out }}=\int_{0}^{\lambda_{0}} p_{\lambda}(\lambda) d \lambda
$$

As an illustrative example, the outage probability of dualhop and triple-hop communication systems can be obtained applying [18]:

$$
\begin{aligned}
P_{\text {out }}= & F_{\lambda_{1}}(\lambda)\left(1-F_{\lambda_{2}}(\lambda)\right)+F_{\lambda_{2}}(\lambda)\left(1-F_{\lambda_{1}}(\lambda)\right) \\
& +F_{\lambda_{1}}(\lambda) F_{\lambda_{2}}(\lambda)
\end{aligned}
$$

$$
\begin{aligned}
P_{\text {out }}= & F_{\lambda_{1}}(\lambda)\left(1-F_{\lambda_{2}}(\lambda)\right)\left(1-F_{\lambda_{3}}(\lambda)\right) \\
& +F_{\lambda_{2}}(\lambda)\left(1-F_{\lambda_{1}}(\lambda)\right)\left(1-F_{\lambda_{3}}(\lambda)\right) \\
& +F_{\lambda_{3}}(\lambda)\left(1-F_{\lambda_{1}}(\lambda)\right)\left(1-F_{\lambda_{2}}(\lambda)\right) \\
& +F_{\lambda_{1}}(\lambda) F_{\lambda_{2}}(\lambda)\left(1-F_{\lambda_{3}}(\lambda)\right) \\
& +F_{\lambda_{1}}(\lambda) F_{\lambda_{3}}(\lambda)\left(1-F_{\lambda_{2}}(\lambda)\right) \\
& +F_{\lambda_{2}}(\lambda) F_{\lambda_{3}}(\lambda)\left(1-F_{\lambda_{1}}(\lambda)\right) \\
& +F_{\lambda_{1}}(\lambda) F_{\lambda_{2}}(\lambda) F_{\lambda_{3}}(\lambda) .
\end{aligned}
$$

The curves representing PDF of $\lambda$ and outage probability of multi-hop (dual-hop and triple-hop) communication system in Nakagami- $m$ fading environment are presented in Figure 2. Without loss of generality, we assumed that the ratios of average powers are equal on all terminals inputs; that is, $\gamma_{i}=\Omega_{x_{i}} \Omega_{z_{i}} / \Omega_{y_{i}} \Omega_{w_{i}}=\gamma, i=\overline{1, N}$. Namely, signal is amplified in terminal so that the ratio of average powers at the input of the next terminal is equal to the ratio of average powers at the input of previous one.

It is noticeable that the outage probability is higher for lower values of Nakagami- $m$ parameter which is connected with signal, that is, in environment with higher fading severity. Increasing power of the useful signal increases values of the $\gamma$ parameter, and as a result we have decline of the outage probability. Also, having in mind that $\gamma_{i}=\gamma, i=\overline{1, N}$, the higher values of $N$ imply larger distance between the source and destination terminals and higher values of the outage probability.

\section{Conclusion}

The PDF and CDF of ratio of product of two random variables $\lambda=x z / y w$ have been derived. Rayleigh, Weibull, Nakagami$m$, and $\alpha-\mu$ statistical models are included in the paper, so that other researchers and engineers could use our results in a wide range of scenarios in many areas of science. An application of these results for the wireless communications community has been described. Namely, presented results can help the designers of wireless communication systems to simulate different wireless environments where fading and shadowing affect desired signal and CCI and readjust system parameters in order to meet the QoS demands. In our future 


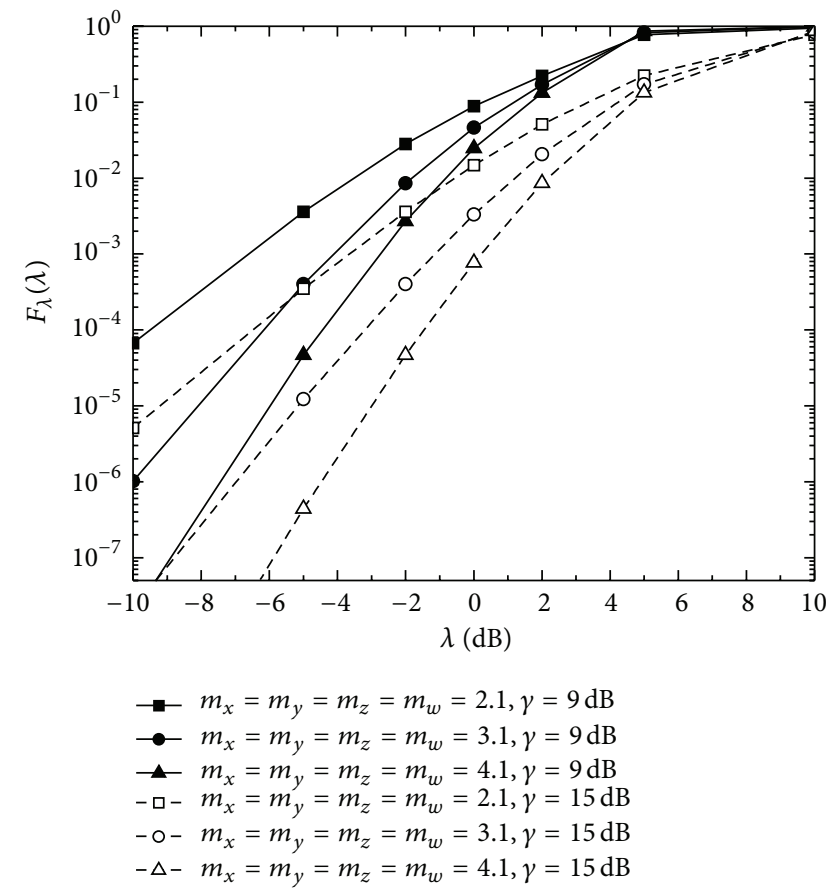

(a)

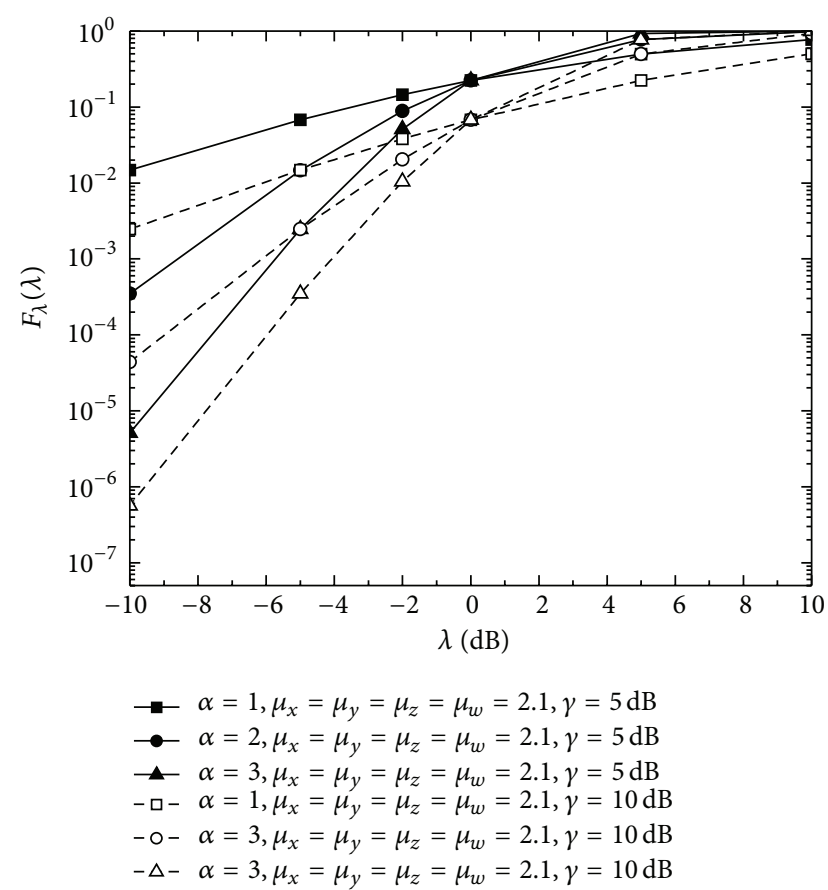

(b)

FIGURE 1: CDF of ratio of products of two $\alpha-\mu$ distribution with special case Nakagami- $m$ distributions when $\alpha=2$.

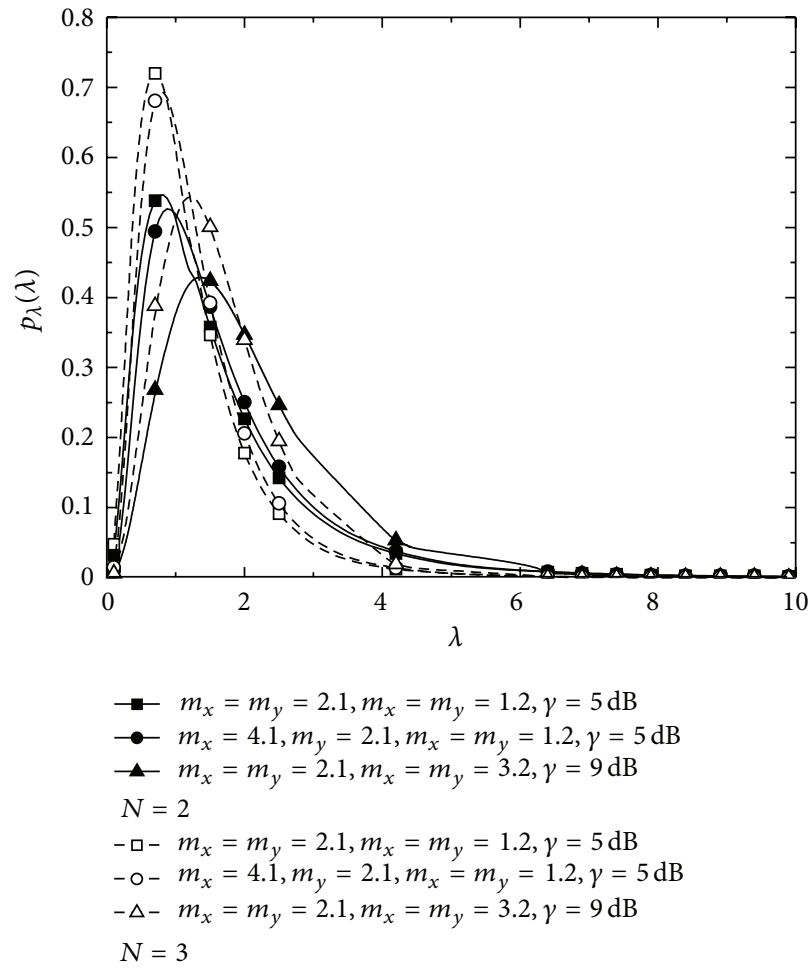

(a)

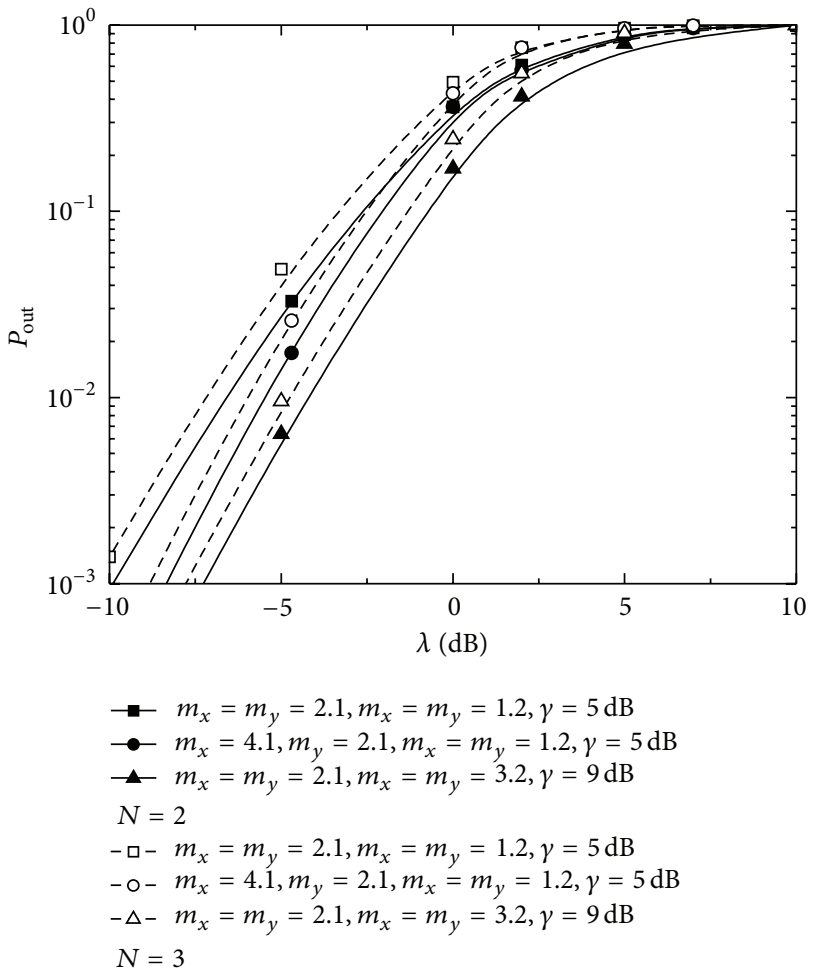

(b)

FIGURE 2: PDF and CDF of ratio of products of two Nakagami- $m$ distributions for dual-and triple-hop communication systems. 
work, we hope to obtain analytical closed form expressions for moment generating functions, amount of fading, capacity of channels, and time of fading for ratio of products of random variables which will have application in analysis of wireless communication systems where signal is affected by fading and shadowing and CCI by fading, signal is affected by fading and CCI by fading and shadowing, and finally where both desired signal and CCI are affected by fading and shadowing simultaneously which is realistic scenario in modern urban areas.

\section{Acknowledgment}

This work has been funded by the Serbian Ministry for Science under the Project III-44006.

\section{References}

[1] A. Melinkov, Cellural Networks: Positioning, Performance Analysis, Reliability, InTech, 2011.

[2] "IEEE Standard-Part 11: Wireless LAN Medium Access Control and Physical Layer Specification," IEEE 802.11, June 2007.

[3] "Bluetooth," http://www.bluetooth.com/bluetooth.

[4] IEFT Mobile Ad Hoc networks (MANET) Working Group, http://www.ietf.org/html.charters/manet-charter.html.

[5] "IEEE Standard for Local and Metropolitan Areas Networks," IEEE 802.16, 2004.

[6] M. K. Simon and M.-S. Alouini, Digital Communication Over Fading Channels, Wiley, New York, NY, USA, 1st edition, 2000.

[7] G. E. Corazza and F. Vatalaro, "Statistical model for land mobile satellite channels and its application to nongeostationary orbit systems," IEEE Transactions on Vehicular Technology, vol. 43, no. 3, pp. 738-742, 1994.

[8] P. G. Babalis and C. N. Capsalis, "Impact of the combined slow and fast fading channel characteristics on the symbol error probability for multipath dispersionless channel characterized by a small number of dominant paths," IEEE Transactions on Communications, vol. 47, no. 5, pp. 653-657, 1999.

[9] J. D. Parsons, The Mobile Radio Propagation Channels, Wiley, New York, NY, USA, 2nd edition, 2000.

[10] M. O. Hasna and M. S. Alouini, "End-to-end performance of transmission systems with relays over Rayleigh-fading channels," IEEE Transactions on Wireless Communications, vol. 2, no. 6, pp. 1126-1131, 2003.

[11] D. Chizhik, J. Ling, P. W. Wolniansky, R. A. Valenzuela, N. Costa, and K. Huber, "Multiple-input-multiple-output measurements and modeling in Manhattan," IEEE Journal on Selected Areas in Communications, vol. 21, no. 3, pp. 321-331, 2003.

[12] F. Babich and G. Lombardi, "Statistical analysis and characterization of the indoor propagation channel," IEEE Transactions on Communications, vol. 48, no. 3, pp. 455-464, 2000.

[13] G. Tzeremes and C. G. Christodoulou, "Use of Weibull distribution for describing outdoor multipath fading," in Proceedings of the IEEE Antennas and Propagation Society International Symposium, pp. 232-235, San Antonio, Tex, USA, June 2002.

[14] M. Nakagami, "The m-distribution-a general formula of intensity distribution of rapid fading," in Statistical Methods in Radio Wave Propagation, pp. 3-36, Pergamon Press, Oxford, UK, 1960.
[15] U. Charash, "Reception through Nakagami fading multipath channels with random delay," IEEE Transactions on Communications, vol. 27, no. 4, pp. 657-670, 1979.

[16] M. D. Yacoub, “The $\alpha-\mu$ distribution: a general fading distribution," Proceedings of the IEEE International Symposium on Personal, Indoor and Mobile Radio Communications (PIMRC '02), vol. 2, pp. 629-633, 2002.

[17] D. B. Da Costa, M. D. Yacoub, and G. Fraidenraich, "Secondorder statistics of equal-gain and maximal-ratio combining for the $\alpha-\mu$ (Generalized Gamma) fading distribution," in Proceedings of the IEEE 9th International Symposium on Spread Spectrum Symposium on Spread Spectrum Techniques and Applications (ISSSTA '06), pp. 342-346, August 2006.

[18] E. Mekić, M. Stefanović, P. Spalević, N. Sekulović, and A. Stanković, "Statistical analysis of ratio of random variables and its application in performance analysis of multihop wireless transmissions," Mathematical Problems in Engineering, vol. 2012, Article ID 841092, 10 pages, 2012.

[19] E. Mekić, N. Sekulović, M. Bandjur, M. Stefanović, and P. Spalević, "The distribution of ratio of random variable and product of two random variables and its application in performance analysis of multi-hop relaying communications over fading channels," Przeglad Elektrotechniczy, vol. 88, pp. 133-137, 2012.

[20] I. S. Gradshteyn and I. M. Ryzhik, Table of Integrals, Series, and Products, Academic Press, New York, NY, USA, 7th edition, 2007.

[21] http://functions.wolfram.com/07.45.26.0005.01.

[22] V. S. Adamchik and O. I. Marichev, "Algorithm for calculating integrals of hypergeometric type functions and its realization in reduce system," in Proceedings of the International Symposium on Symbolic and Algebraic Computation (ISSAC '90), pp. 212224, August 1990.

[23] G. K. Karagiannidis, T. A. Tsiftsis, and R. K. Mallik, "Bounds for multihop relayed communications in Nakagami-m fading," IEEE Transactions on Communications, vol. 54, no. 1, pp. 18-22, 2006.

[24] M. O. Hasna and M. S. Alouini, "Outage probability of multihop transmission over Nakagami fading channels," IEEE Communications Letters, vol. 7, no. 5, pp. 216-218, 2003.

[25] C. Zhong, S. Jin, and K. K. Wong, "Outage probability of dual-hop relay channels in the presence of interference," in Proceedings of the IEEE 69th Vehicular Technology Conference (VTC '09), pp. 1-5, April 2009. 


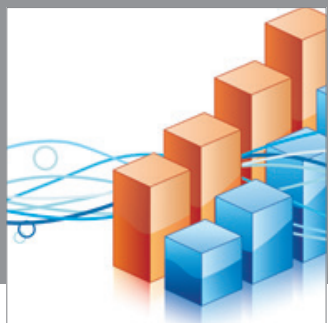

Advances in

Operations Research

mansans

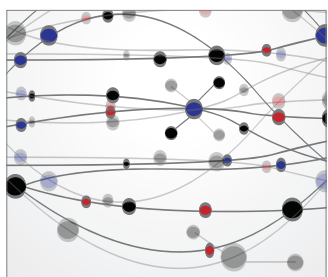

The Scientific World Journal
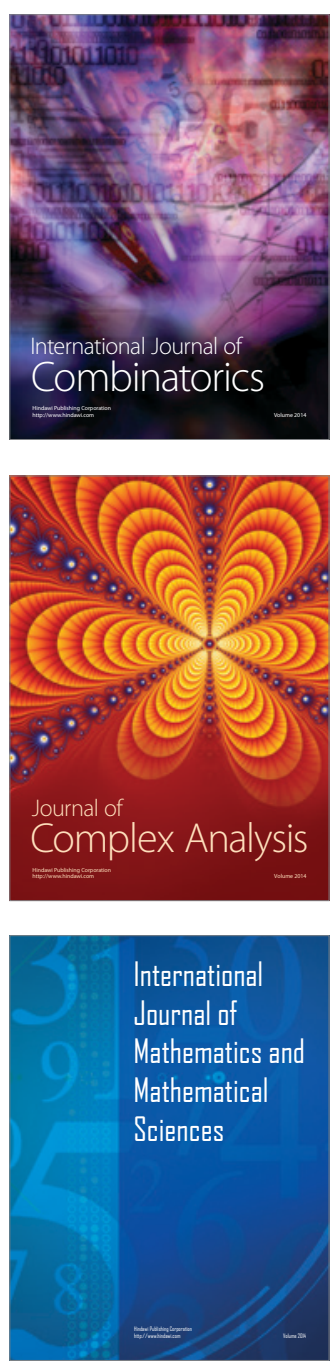
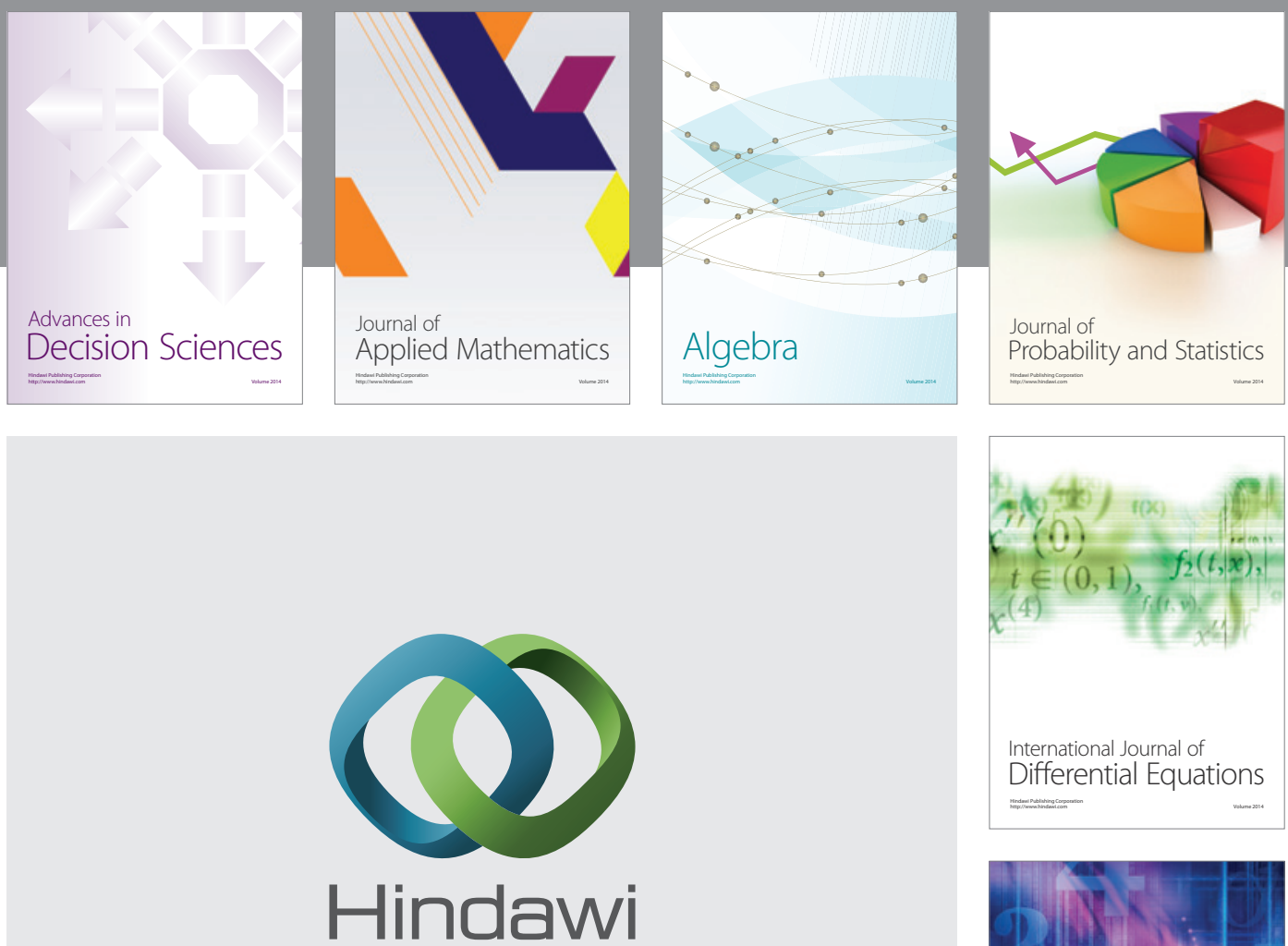

Submit your manuscripts at http://www.hindawi.com
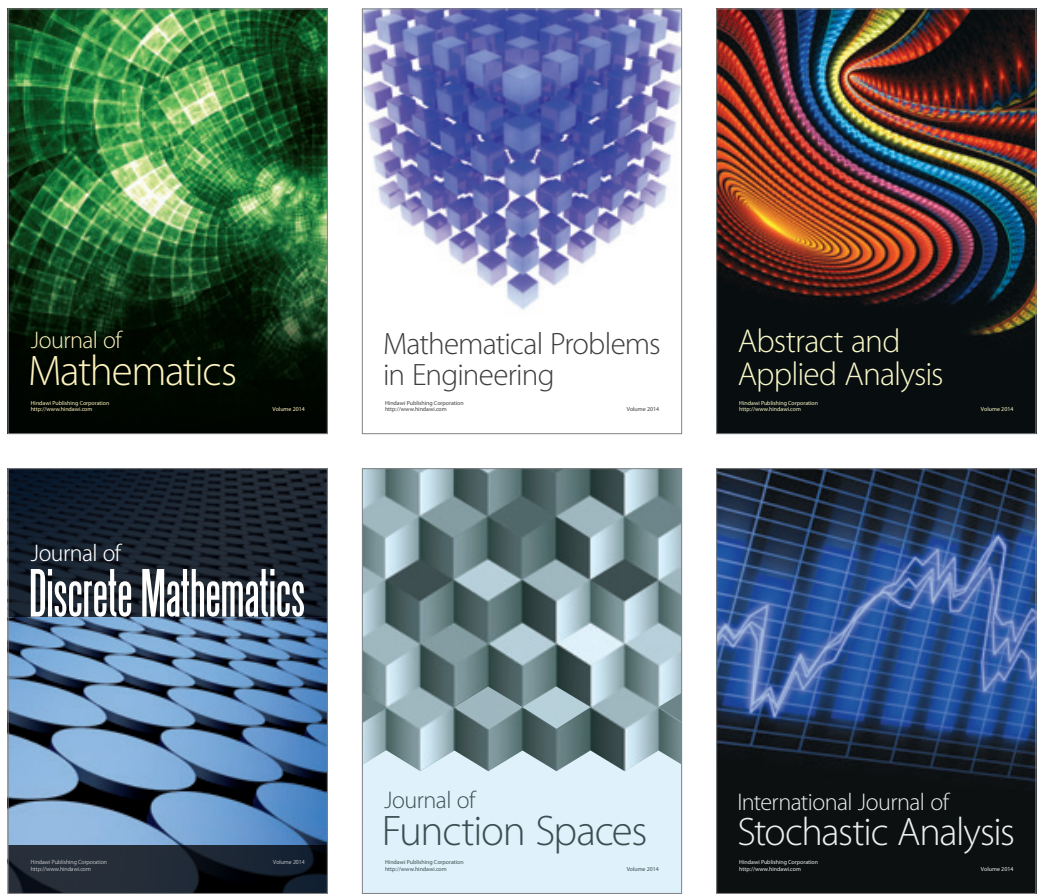

Journal of

Function Spaces

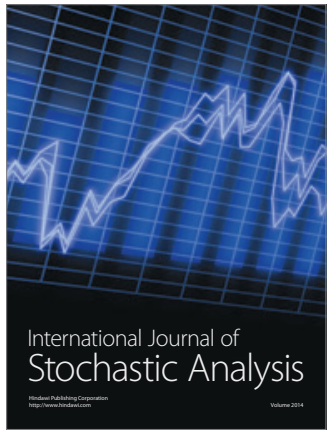

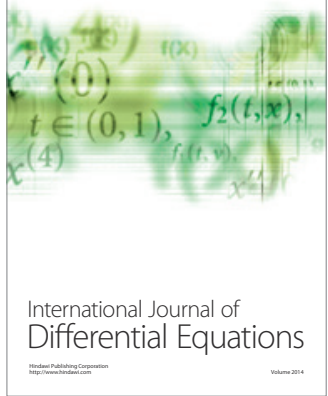
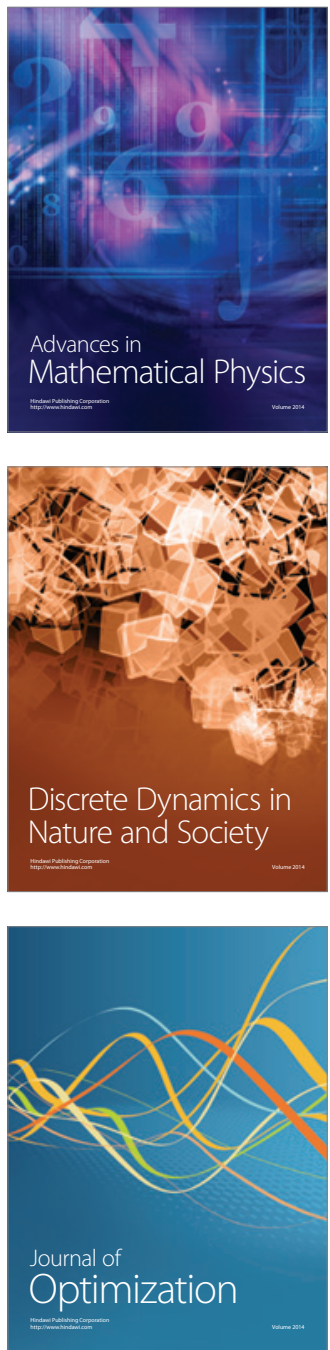